\title{
Proofs of two conjectures of Kenyon and Wilson on Dyck tilings
}

\author{
Jang Soo Kim \\ School of Mathematics, University of Minnesota, USA.
}

\begin{abstract}
Recently, Kenyon and Wilson introduced a certain matrix $M$ in order to compute pairing probabilities of what they call the double-dimer model. They showed that the absolute value of each entry of the inverse matrix $M^{-1}$ is equal to the number of certain Dyck tilings of a skew shape. They conjectured two formulas on the sum of the absolute values of the entries in a row or a column of $M^{-1}$. In this paper we prove the two conjectures. As a consequence we obtain that the sum of the absolute values of all entries of $M^{-1}$ is equal to the number of complete matchings. We also find a bijection between Dyck tilings and complete matchings.

Résumé. Récemment, Kenyon et Wilson ont introduit une certaine matrice $M$ afin de calculer des probabilités d'appariement dans ce qu'ils appellent le modèle double-dimère. Ils ont montré que la valeur absolue de chaque entrée de la matrice inverse $M^{-1}$ est égal au nombre de pavages de Dyck d'une certaine forme gauche. Ils ont conjecturé deux formules sur la somme des valeurs absolues des entrées dans une rangée ou une colonne de $M^{-1}$. Dans cet article, nous prouvons les deux conjectures. En conséquence on obtient que la somme des valeurs absolues de toutes les entrées de $M^{-1}$ est égale au nombre de couplages parfaits. Nous trouvons aussi une bijection entre les pavages de Dyck et les couplages parfaits.
\end{abstract}

Keywords: Dyck paths, Dyck tilings, matchings, Hermite histories, orthogonal polynomials

\section{Introduction}

A Dyck path of length $2 n$ is a lattice path from $(0,0)$ to $(n, n)$ consisting of up steps $(0,1)$ and down steps $(1,0)$ that never goes below the line $y=x$. The set of Dyck paths of length $2 n$ is denoted Dyck $(2 n)$. In this paper we will sometimes identify a Dyck path $\lambda$ with a partition as shown in Figure 1 . For $\lambda, \mu \in \operatorname{Dyck}(2 n)$, if $\mu$ is above $\lambda$, then the skew shape $\lambda / \mu$ is well defined.
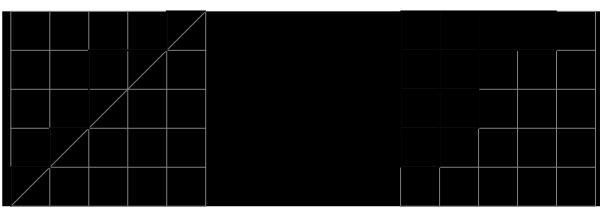

Figure 1: A Dyck path identified with the partition $(4,2,2,1)$. 
For two Dyck paths $\lambda$ and $\mu$, we define $\lambda \succ \mu$ if $\lambda$ can be obtained from $\mu$ by choosing some matching pairs of up steps and down steps and exchanging the chosen up steps and down steps, see Figure 2 In order to compute pairing probabilities of so-called the double-dimer model, Kenyon and Wilson [7, 8] introduced a matrix $M$ defined as follows. The rows and columns of $M$ are indexed by $\lambda, \mu \in \operatorname{Dyck}(2 n)$, and $M_{\lambda, \mu}=1$ if $\lambda \succ \mu$, and $M_{\lambda, \mu}=0$ otherwise.

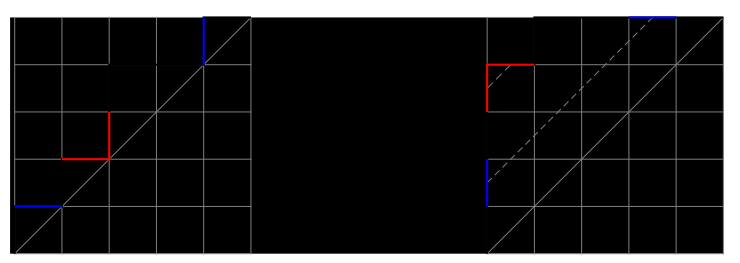

Figure 2: An example of the order $\succ$ on Dyck paths.

A ribbon is a connected skew shape which does not contain a $2 \times 2$ box. A Dyck tile is a ribbon such that the centers of the cells form a Dyck path. The length of a Dyck tile is the length of the Dyck path obtained by joining the centers of the cells, see Figure 3 .

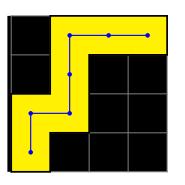

Figure 3: A Dyck tile of length 6.

For $\lambda, \mu \in \operatorname{Dyck}(2 n)$, a (cover-inclusive) Dyck tiling of $\lambda / \mu$ is a tiling with Dyck tiles such that for two Dyck tiles $T_{1}$ and $T_{2}$, if $T_{1}$ has a cell to the southeast of a cell of $T_{2}$, then a southeast translation of $T_{2}$ is contained in $T_{1}$. We denote by $\mathcal{D}(\lambda / \mu)$ the set of Dyck tilings of $\lambda / \mu$. Kenyon and Wilson [8, Theorem 1.5] showed that the inverse matrix $M^{-1}$ of $M$ can be expressed using Dyck tilings:

$$
M_{\lambda, \mu}^{-1}=(-1)^{|\lambda / \mu|} \times|\mathcal{D}(\lambda / \mu)|,
$$

where $|\lambda / \mu|$ denote the number of cells in $\lambda / \mu$.

In this paper we prove two conjectures of Kenyon and Wilson on $q$-analogs of the sum of the absolute values of the entries in a row or a column of $M^{-1}$. In order to state the conjectures we need the following notions.

A chord of a Dyck path $\lambda$ is a matching pair of an up step and a down step. We denote by $\operatorname{Chord}(\lambda)$ the set of chords of $\lambda$. For $c \in \operatorname{Chord}(\lambda)$, the length $|c|$ of $c$ is defined to be the difference between the $x$-coordinates of the starting point of the up step and the ending point of the down step. The height ht $(c)$ of $c$ is defined to be $i$ if $c$ is between $y=x+i-1$ and $y=x+i$. See Figure 4 for an example.

We use the standard notations for $q$-integers:

$$
\begin{aligned}
{[n]_{q} } & =1+q+\cdots+q^{n-1}, \\
{[n]_{q} ! } & =[1]_{q}[2]_{q} \cdots[n]_{q} .
\end{aligned}
$$



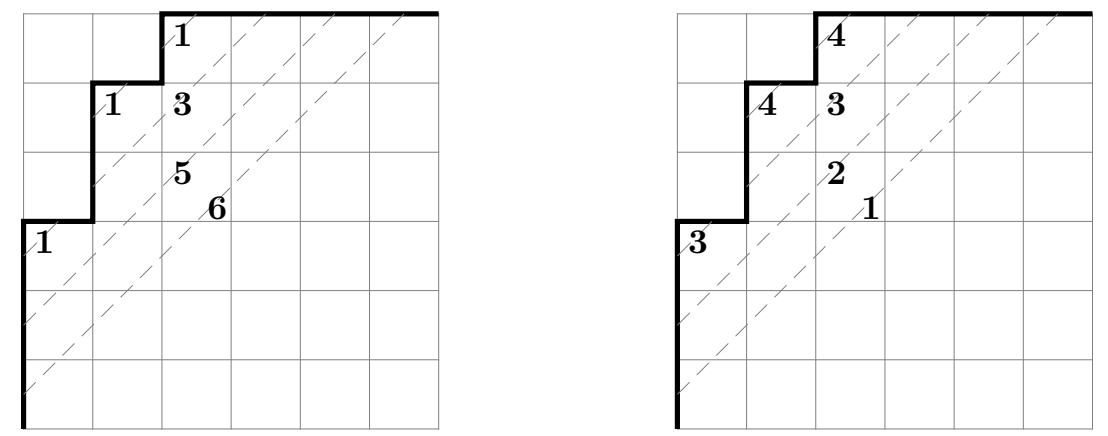

Figure 4: The lengths (left) and the heights (right) of the chords of a Dyck path.

Also, we denote $[n]=\{1,2, \ldots, n\}$, which should not be confused with the $q$-integers.

We now state the main theorems.

Theorem 1.1. [8. Conjecture 1] Given a Dyck path $\lambda \in \operatorname{Dyck}(2 n)$, we have

$$
\sum_{\mu \in \operatorname{Dyck}(2 n)} \sum_{T \in \mathcal{D}(\lambda / \mu)} q^{(|\lambda / \mu|+|T|) / 2}=\frac{[n]_{q} !}{\prod_{c \in \operatorname{Chord}(\lambda)}[|c|]_{q}} .
$$

Theorem 1.2. [8. Conjecture 2] Given a Dyck path $\mu \in \operatorname{Dyck}(2 n)$, we have

$$
\sum_{\lambda \in \operatorname{Dyck}(2 n)} \sum_{T \in \mathcal{D}(\lambda / \mu)} q^{|T|}=\prod_{c \in \operatorname{Chord}(\mu)}[\operatorname{ht}(c)]_{q} .
$$

Our proof of Theorem 1.2 is simpler than the proof of Theorem 1.1 . So we will first present the proof of Theorem 1.2 .

The rest of the paper is organized as follows. In Section 2 we prove Theorem 1.2. In Section 3 we introduce truncated Dyck tilings, which are very similar to Dyck tilings, and some properties of them. We then state a generalization of Theorem 1.1. In Section 4 we construct a bijection between Dyck tilings and complete matchings, and discuss some applications of the bijection.

This is an extended abstract of [9].

\section{Proof of Theorem 1.2}

We denote by $\delta_{n-1}$ the staircase partition $(n-1, n-2, \ldots, 1)$. Note that if $\mu \in \operatorname{Dyck}(2 n)$, we have $\mu \subset \delta_{n-1}$.

We will prove Theorem 1.2 by induction on the number $m(\mu)$ of cells in $\left|\delta_{n-1} / \mu\right|$, where $n$ is the half-length of $\mu$. If $m(\mu)=0$, then $\mu=\delta_{n-1}$ and the theorem is clear. Assume $m \geq 1$ and the theorem is true for all $\nu$ with $m(\nu)<m$. Now suppose $\mu \in \operatorname{Dyck}(2 n)$ with $m(\mu)=m$. Since $\left|\delta_{n-1} / \mu\right|=m \geq 1$, we can pick a cell $s \in \delta_{n-1} / \mu$ such that $\mu \cup s$ is also a partition. Let $h$ be the height of the chord of $\mu$ contained in $s$. Consider a tiling $T \in \mathcal{D}(\lambda / \mu)$ for some $\lambda \in \operatorname{Dyck}(2 n)$. Then there are two cases. 
Case 1: $s$ by itself is a tile in $T$. Let $\mu^{\prime}=\mu \cup\{s\}$. Then $T^{\prime}=T \backslash\{s\}$ is a tiling in $\mathcal{D}\left(\lambda / \mu^{\prime}\right)$. Thus the sum of $q^{|T|}$ for all such choices of $\lambda$ and $T$ equals

$$
\sum_{\lambda \in \operatorname{Dyck}(2 n)} \sum_{T^{\prime} \in \mathcal{D}\left(\lambda / \mu^{\prime}\right)} q^{\left|T^{\prime}\right|+1}
$$

By the induction hypothesis, the above sum is equal to

$$
q \prod_{c \in \operatorname{Chord}\left(\mu^{\prime}\right)}[\operatorname{ht}(c)]_{q}=\frac{q[h-1]_{q}}{[h]_{q}} \prod_{c \in \operatorname{Chord}(\mu)}[\operatorname{ht}(c)]_{q},
$$

where we use the fact that $\mu^{\prime}$ has one more chord of height $h-1$ and one less chord of height $h$ than $\mu$, see Figure 5
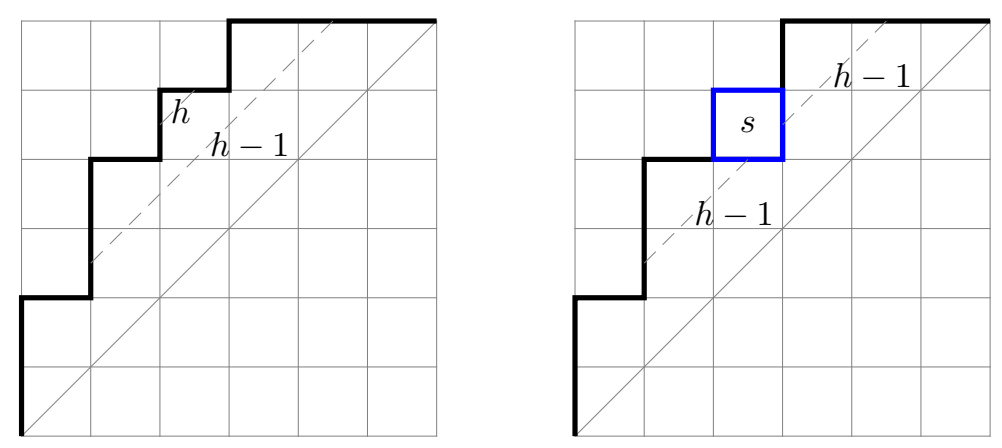

Figure 5: $\mu \cup\{s\}$ has one more chord of height $h-1$ and one less chord of height $h$ than $\mu$.

Case 2: Otherwise we have either $s \notin \lambda / \mu$ or $s$ is covered by a Dyck tile of length greater than 0 . Then we collapse the slice containing $s$, in other words, remove the region in $\lambda / \mu$ bounded by the two lines with slope -1 passing through the northeast corner and the southwest corner of $s$ and attach the two remaining regions, see Figure 6 Let $\lambda^{\prime}, \mu^{\prime}$, and $T^{\prime}$ be the resulting objects obtained from $\lambda$, $\mu$, and $T$ in this way. Since the collapsed slice is completely covered by Dyck tiles of length greater than 0 , the original objects $\lambda, \mu$, and $T$ can be obtained from $\lambda^{\prime}, \mu^{\prime}$, and $T^{\prime}$. We also have $|T|=\left|T^{\prime}\right|$. Thus, by the induction hypothesis, the sum of $q^{|T|}$ for all possible choices of $\lambda$ and $T$ is equal to

$$
\sum_{\lambda^{\prime} \in \operatorname{Dyck}(2 n)} \sum_{T^{\prime} \in \mathcal{D}\left(\lambda^{\prime} / \mu^{\prime}\right)} q^{\left|T^{\prime}\right|}=\prod_{c \in \operatorname{Chord}\left(\mu^{\prime}\right)}[\operatorname{ht}(c)]_{q}=\frac{1}{[h]_{q}} \prod_{c \in \operatorname{Chord}(\mu)}[\operatorname{ht}(c)]_{q} .
$$

Summing (11) and (2), the theorem is also true for $\nu$. By induction, the theorem is proved.

We note that the proof in this section was also discovered independently by Matjaž Konvalinka (personal communication with Matjaž Konvalinka).

It is not difficult to construct a bijection between Dyck tilings and Hermite histories (see Section for the definition) by the same recursive manner as in the proof in this section. In fact, the bijection obtained in this way has a non-recursive description, which we will present in Section 4 


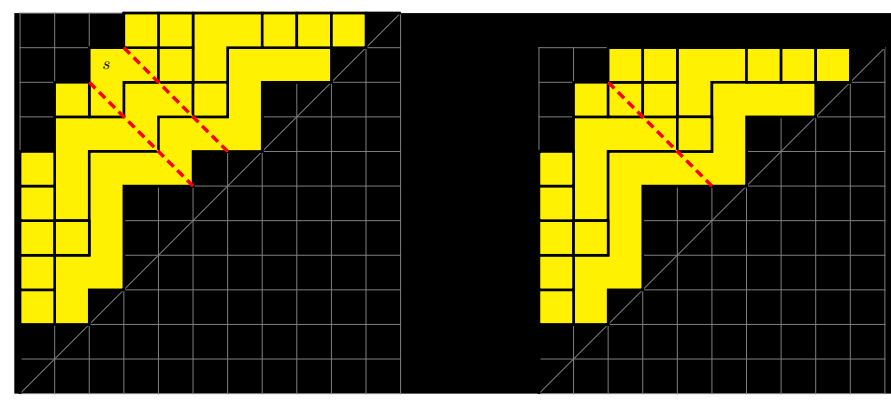

Figure 6: Collapsing the slice containing $s$.

\section{Truncated Dyck tilings}

In this section we state a generalization of Theorem 1.1 . We first need to reformulate Theorem 1.1 .

For a Dyck tiling $T$ we define $\|T\|$ to be the sum of the half-lengths of all Dyck tiles in $T$.

Lemma 3.1. For $T \in \mathcal{D}(\lambda / \mu)$, we have

$$
q^{(|\lambda / \mu|+|T|) / 2}=q^{|\lambda / \mu|-\|T\|} .
$$

By Lemma 3.1, we can rewrite Theorem 1.1 as follows.

Theorem 3.2. Given a Dyck path $\lambda \in \operatorname{Dyck}(2 n)$, we have

$$
\sum_{\mu \in \operatorname{Dyck}(2 n)} \sum_{T \in \mathcal{D}(\lambda / \mu)} q^{|\lambda / \mu|-\|T\|}=\frac{[n]_{q} !}{\prod_{c \in \operatorname{Chord}(\lambda)}[|c|]_{q}} .
$$

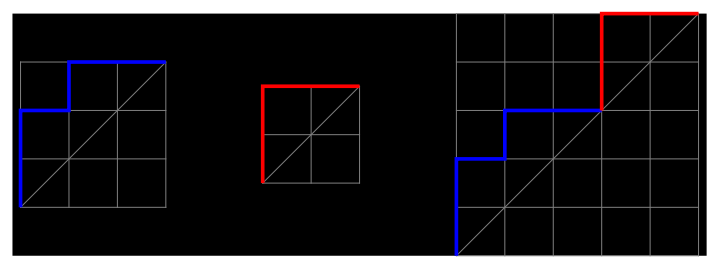

Figure 7: The $*$ operation on two Dyck paths.

For two Dyck paths $\lambda$ and $\mu$ (not necessarily of the same length) we define $\lambda * \mu$ to be the Dyck path obtained from $\lambda$ by attaching $\mu$ at the end of $\lambda$, see Figure 7 For a nonnegative integer $k$, we denote by $\Delta_{k}$ the Dyck path of length $2 k$ consisting of $k$ consecutive up steps and $k$ consecutive down steps. For nonnegative integers $k_{1}, \ldots, k_{r}$, we define

$$
\Delta_{k_{1}, \ldots, k_{r}}=\Delta_{k_{1}} * \cdots * \Delta_{k_{r}}
$$

For an object $X$, which may be a point, a lattice path, or a tile, we denote by $X+(i, j)$ the translation of $X$ by $(i, j)$. So far, we have only considered $\lambda / \mu$ for two Dyck paths $\lambda$ and $\mu$ starting and ending at the same points. We extend this definition as follows. 
Suppose $\lambda$ is a Dyck path from $O=(0,0)$ to $N=(n, n)$ and $\mu$ is a lattice path from $P=O+(-a, a)$ to $Q=N+(-b, b)$ for some nonnegative integers $a$ and $b$ such that $\mu$ never goes below $\lambda$. Then we define $\lambda / \mu$ to be the region bounded by $\lambda, \mu$, and the segments $O P$ and $N Q$. We denote by $|\lambda / \mu|$ the area of the region $\lambda / \mu$. Note that this notation is consistent with the number $|\lambda / \mu|$ of cells of $\lambda / \mu$ when $\lambda / \mu$ is a skew shape. Given $\lambda, a$, and $b$, we denote by $L(\lambda ; a, b)$ the set of all lattice paths from $P$ to $Q$ which never go below $\lambda$.

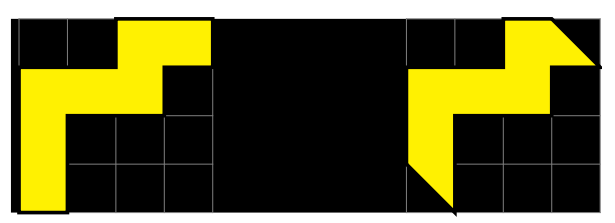

Figure 8: A Dyck tile and the corresponding truncated Dyck tile.

Definition 1. A truncated Dyck tile is a tile obtained from a Dyck tile of positive length by cutting off the northeast half-cell and the southwest half-cell as shown in Figure 8. A (cover-inclusive) truncated Dyck tiling of a region $\lambda / \mu$ is a tiling $T$ of a sub-region of $\lambda / \mu$ with truncated Dyck tiles satisfying the following conditions:

- For each tile $\eta \in T$, if $(\eta+(1,-1)) \cap \lambda / \mu \neq \emptyset$, then there is another tile $\eta^{\prime} \in T$ containing $(\eta+(1,-1))$.

- There are no two tiles sharing a border with slope -1 .

Let $\mathcal{T} \mathcal{D}(\lambda / \mu)$ denote the set of truncated Dyck tilings of $\lambda / \mu$.

If $\mu \in L(\lambda ; 0,0)$, there is a natural bijection between $\mathcal{D}(\lambda / \mu)$ and $\mathcal{T} \mathcal{D}(\lambda / \mu)$ as follows. For every tile in $T \in \mathcal{D}(\lambda / \mu)$, remove the northeast half-cell and the southwest half-cell as shown in Figure 9 Note that the Dyck tiles of length 0 simply disappear.
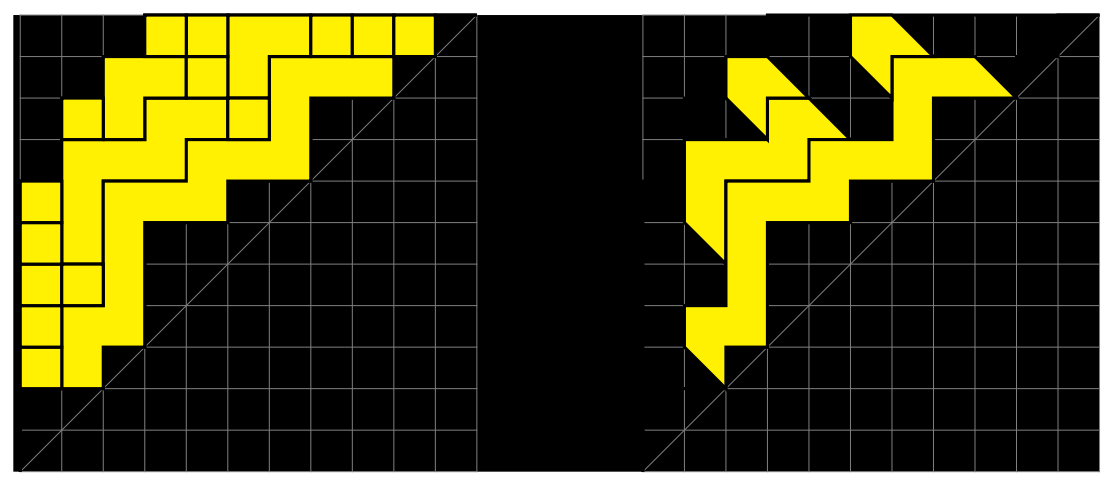

Figure 9: A Dyck tiling and the corresponding truncated Dyck tiling. 
Let

$$
B_{q}(\lambda ; a, b)=\sum_{\mu \in L(\lambda ; a, b)} \sum_{T \in \mathcal{T} \mathcal{D}(\lambda / \mu)} q^{|\lambda / \mu|-\|T\|} .
$$

Note that $B_{q}(\lambda ; a, b)$ is not necessarily a polynomial in $q$, but a polynomial in $q^{1 / 2}$. In fact $B_{q}(\lambda ; a, b)$ is a polynomial in $q$ if and only if $a \equiv b \bmod 2$.

We now state a generalization of Theorem 3.2 . or equivalently, Theorem 1.1 .

Theorem 3.3. For $\lambda \in \operatorname{Dyck}(2 n)$, nonnegative integers $a$ and $b$, we have

$$
B_{q}(\lambda ; a, b)=\frac{[n]_{q} !}{\prod_{c \in \operatorname{Chord}(\lambda)}[|c|]_{q}} B_{q}\left(\Delta_{n} ; a, b\right) .
$$

Note that if $a=b=0$ in Theorem 3.3 , we obtain Theorem 3.2. We will only outline the proof of Theorem 3.3 which consists of three steps. In the first step we prove the theorem in the case $\lambda=\Delta_{n_{1}, \ldots, n_{k}}$ and $a=b=0$. In the second step we prove theorem in the case $\lambda=\Delta_{n_{1}, \ldots, n_{k}}$, and $a$ and $b$ are arbitrary. In the third step we prove the theorem without restrictions.

\section{A bijection from Dyck tilings to matchings}

In this section we find a bijection sending Dyck tilings to Hermite histories, which are in simple bijection with complete matchings. We start by defining these objects.

A (complete) matching on $[2 n]$ is a set of pairs $(i, j)$ of integers in $[2 n]$ with $i<j$ such that each integer in $[2 n]$ appears exactly once. We denote by $\mathcal{M}(2 n)$ the set of matchings on $[2 n]$. It is convenient to represent $\pi \in \mathcal{M}(2 n)$ by the diagram obtained by joining $i$ and $j$ with an arc for each $(i, j) \in \pi$ as shown in Figure 10. We define the shape of $\pi$ to be the Dyck path such that the $i$ th step is an up step if $(i, j) \in \pi$ for some $j$, and a down step otherwise, see Figure 10 For a Dyck path $\mu$, the set of matchings with shape $\mu$ is denoted by $\mathcal{M}(\mu)$. A crossing (resp. nesting) of $\pi \in \mathcal{M}(2 n)$ is a set of two pairs $(i, j)$ and $\left(i^{\prime}, j^{\prime}\right)$ in $\pi$ such that $i<i^{\prime}<j<j^{\prime}$ (resp. $i<i^{\prime}<j^{\prime}<j$ ). The number of crossings (resp. nestings) of $\pi$ is denoted by $\operatorname{cr}(\pi)$ (resp. ne $(\pi)$ ). For example, if $\pi$ is the matching in Figure 10, we have $\operatorname{cr}(\pi)=2$ and $\operatorname{ne}(\pi)=1$.
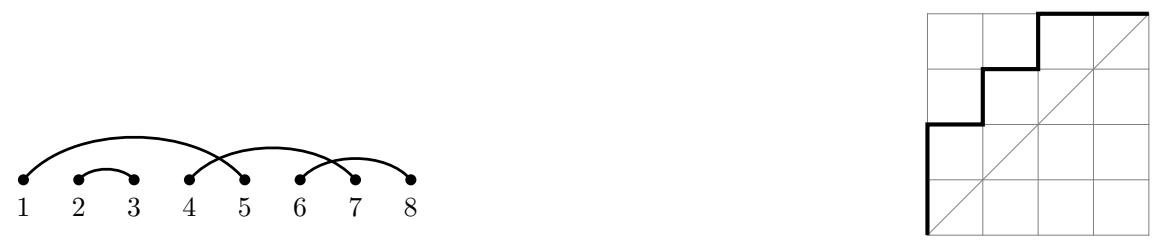

Figure 10: The diagram (left) of the matching $\{(1,5),(2,3),(4,7),(6,8)\}$ and its shape (right).

A Hermite history of length $2 n$ is a pair $(\mu, H)$ of a Dyck path $\mu \in \operatorname{Dyck}(2 n)$ and a labeling $H$ of the down steps of $\mu$ such that the label of a down step of height $h$ is an integer in $\{0,1, \ldots, h-1\}$. We denote by $\mathcal{H}(2 n)$ the set of Hermite histories of length $2 n$, and by $\mathcal{H}(\mu)$ the set of Hermite histories with Dyck path $\mu$. There is a well-known bijection $\zeta: \mathcal{M}(2 n) \rightarrow \mathcal{H}(2 n)$, see [17] or [6]. For $\pi \in \mathcal{M}(2 n)$, the 
corresponding Hermite history $\zeta(\pi)=(\mu, H)$ is defined as follows. The Dyck path $\mu$ is the shape of $\pi$. For a down step $D$ of $\mu$, if it is the $j$ th step, there is a pair $(i, j) \in \pi$. Then the label of $D$ is defined to be the number of pairs $\left(i^{\prime}, j^{\prime}\right) \in \pi$ such that $i<i^{\prime}<j<j^{\prime}$. For example, if $\pi$ is the matching in Figure 10 . then $\mu$ is the Dyck path in Figure 10 and the labels of the downs steps are 0, 1, 1, and 0 in this order. Note that $\zeta$ is also a bijection from $\mathcal{M}(\mu)$ to $\mathcal{H}(\mu)$.

For $\mu \in \operatorname{Dyck}(2 n)$ and $(\mu, H) \in \mathcal{H}(2 n)$, we define

$$
\begin{aligned}
\operatorname{ht}(\mu) & =\sum_{c \in \operatorname{Chord}(\mu)}(\operatorname{ht}(c)-1), \\
\|H\| & =\sum_{i \in H} i .
\end{aligned}
$$

The next lemma easily follows from the construction of the map $\zeta$.

Lemma 4.1. Let $\zeta(\pi)=(\mu, H)$. Then $\|H\|=\operatorname{cr}(\pi)$ and $\operatorname{ht}(\mu)=\operatorname{cr}(\pi)+\operatorname{ne}(\pi)$.

From now on we will use the following notations: for $\lambda, \mu \in \operatorname{Dyck}(2 n)$,

$$
\begin{aligned}
& \mathcal{D}(\lambda / *)=\bigcup_{\nu \in \operatorname{Dyck}(2 n)} \mathcal{D}(\lambda / \nu), \\
& \mathcal{D}(* / \mu)=\bigcup_{\nu \in \operatorname{Dyck}(2 n)} \mathcal{D}(\nu / \mu), \\
& \mathcal{D}(2 n)=\bigcup_{\nu, \rho \in \operatorname{Dyck}(2 n)} \mathcal{D}(\nu / \rho) .
\end{aligned}
$$

For a Dyck tile $\eta$, we define the entry (resp. exit) of $\eta$ to be the north border (resp. the south border) of the northeast cell (resp. the southwest cell) of $\eta$.

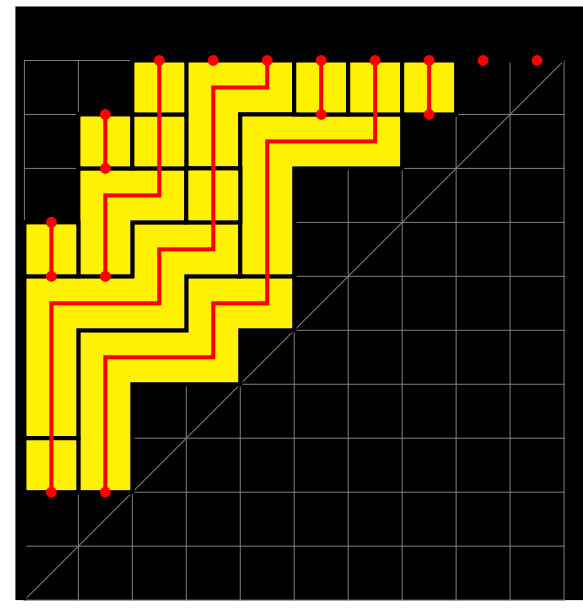

Figure 11: An example of the map $\psi$. 
For $T \in \mathcal{D}(* / \mu)$, we define $\psi(T)=(\mu, H)$ as follows. The label of a down step $s$ of $\mu$ is the number of Dyck tiles that we pass in the following process. We start from $s$ and travel to the south until we reach a border that is not an entry; if we arrive at the entry of a Dyck tile, then continue traveling from the exit of the Dyck tile, see Figure 11. Observe that every tile is traveled exactly once, which can be checked using the definition of a truncated Dyck tiling. Thus we have $|T|=\|H\|$. It is easy to see that the map $\psi$ has the same recursive structure as the proof of Theorem 1.2 in Section 2 Thus $\psi: \mathcal{D}(* / \mu) \rightarrow \mathcal{H}(\mu)$ is a bijection. It is also possible to construct the inverse map of $\psi$, but it is more complicated than $\psi$.

Theorem 4.2. Given a Dyck path $\mu \in \operatorname{Dyck}(2 n)$, the map $\psi$ gives a bijection $\psi: \mathcal{D}(* / \mu) \rightarrow \mathcal{H}(\mu)$ such that if $\psi(T)=(\mu, H)$, then $|T|=\|H\|$. Thus, $\zeta^{-1} \circ \psi: \mathcal{D}(* / \mu) \rightarrow \mathcal{M}(\mu)$ is a bijection such that if $\left(\zeta^{-1} \circ \psi\right)(T)=\pi$, then $|T|=\operatorname{cr}(\pi)$.

We now discuss several applications of Theorem 4.2 First of all, since

$$
\sum_{H \in \mathcal{H}(\mu)} q^{\|H\|}=\prod_{c \in \operatorname{Chord}(\mu)}[\operatorname{ht}(c)]_{q},
$$

Theorem 4.2 gives a bijective proof of Theorem 1.2

By Theorem 4.2, $\mathcal{D}(2 n), \mathcal{H}(2 n)$, and $\mathcal{M}(2 n)$ have the same cardinality $(2 n-1) ! !=1 \cdot 3 \cdots(2 n-1)$. Therefore, we have

$$
|\mathcal{D}(2 n)|=(2 n-1) ! !
$$

which was also conjectured by Kenyon and Wilson (private communication with David Wilson).

For $T \in \mathcal{D}(\lambda / \mu)$, we define ht $(T)=\operatorname{ht}(\mu)$.

Corollary 4.3. We have

$$
\sum_{T \in \mathcal{D}(2 n)} p^{\mathrm{ht}(T)-|T|} q^{|T|}=\sum_{\pi \in \mathcal{M}(2 n)} p^{\mathrm{ne}(\pi)} q^{\mathrm{cr}(\pi)} .
$$

By Flajolet's theory on continued fractions [3], we have

$$
\sum_{n \geq 0} D_{n}(p, q) x^{n}=\frac{1}{1-\frac{[1]_{p, q} x}{1-\frac{[2]_{p, q} x}{1-\cdots}}}
$$

where $[n]_{p, q}=p^{n-1}+p^{n-2} q+\cdots+p q^{n-2}+q^{n-1}$. By Viennot's theory [16, 17], $D_{n}(p, q)$ is equal to the $2 n$th moment of the orthogonal polynomial $H_{n}(x ; p, q)$ defined by $H_{-1}(x ; p, q)=0, H_{0}(x ; p, q)=1$, and the three term recurrence

$$
H_{n+1}(x ; p, q)=x H_{n}(x ; p, q)-[n]_{p, q} H_{n-1}(x ; p, q) .
$$

In particular, $H_{n}(x ; 1, q)$ is the continuous $q$-Hermite polynomial and $H_{n}\left(x ; q, q^{2}\right)$ is the discrete $q$ Hermite polynomial, see [4, 14]. There are known formulas for the $2 n$th moments of $H_{n}(x ; 1, q)$ and $H_{n}\left(x ; q, q^{2}\right)$. For the $2 n$th moment of $H_{n}(x ; 1, q)$, we have the Touchard-Riordan formula which has various proofs, see [2, 5, 6, 11, 12, 13, 15]:

$$
\sum_{\pi \in \mathcal{M}(2 n)} q^{\operatorname{cr}(\pi)}=\frac{1}{(1-q)^{n}} \sum_{k=0}^{n}\left(\left(\begin{array}{c}
2 n \\
n-k
\end{array}\right)-\left(\begin{array}{c}
2 n \\
n-k-1
\end{array}\right)\right)(-1)^{k} q^{\left(\begin{array}{c}
k+1 \\
2
\end{array}\right) .}
$$


For the $2 n$th moment of $H_{n}\left(x ; q, q^{2}\right)$, we have the following formula, see [4, Proof of Corollary 2] or [14, (5.4)]:

$$
\sum_{\pi \in \mathcal{M}(2 n)} q^{2 \operatorname{cr}(\pi)+\mathrm{ne}(\pi)}=[2 n-1]_{q} ! !
$$

where $[2 n-1]_{q} ! !=[1]_{q}[3]_{q} \cdots[2 n-1]_{q}$.

By Corollary 4.3. (4) and (5) we obtain the following corollary.

Corollary 4.4. We have

$$
\begin{gathered}
\sum_{T \in \mathcal{D}(2 n)} q^{|T|}=\frac{1}{(1-q)^{n}} \sum_{k=0}^{n}\left(\left(\begin{array}{c}
2 n \\
n-k
\end{array}\right)-\left(\begin{array}{c}
2 n \\
n-k-1
\end{array}\right)\right)(-1)^{k} q^{\left(\begin{array}{c}
k+1 \\
2
\end{array}\right)}, \\
\sum_{T \in \mathcal{D}(2 n)} q^{\operatorname{ht}(T)+|T|}=[2 n-1]_{q} ! !
\end{gathered}
$$

\section{Final remarks}

We can generalize the matrix $M$ in the introduction as follows. The matrix $M(p, q)$ is defined by

$$
M(p, q)_{\lambda, \mu}= \begin{cases}p^{|\lambda / \mu|} q^{d(\lambda, \mu)}, & \text { if } \lambda \succ \mu ; \\ 0, & \text { otherwise }\end{cases}
$$

where $d(\lambda, \mu)$ is the number of reversed matching pairs when going from $\mu$ to $\lambda$. Then $M=M(1,1)$. Recall that Kenyon and Wilson [8, Theorem 1.5] proved that

$$
M_{\lambda, \mu}^{-1}=(-1)^{|\lambda / \mu|} \times|\mathcal{D}(\lambda / \mu)| .
$$

It is not hard to see that the proof of the above identity in [8] also implies the following identity, which was first observed by Matjaž Konvalinka (personal communication with Matjaž Konvalinka):

$$
M(p, q)_{\lambda, \mu}^{-1}=\sum_{T \in \mathcal{D}(\lambda / \mu)}(-p)^{|\lambda / \mu|} q^{|T|} .
$$

Note that Theorem 1.1 (resp. Theorem 1.2) is a formula for the sum of the absolute values of the entries in a row of $M\left(q^{1 / 2}, q^{1 / 2}\right)$ (resp. a column of $M(1, q)$ ). Such a sum using $M(p, q)$ does not factor nicely, so it seems difficult to find a formula for the sum.

In Section 4 we have found a bijection $\psi: \mathcal{D}(* / \mu) \rightarrow \mathcal{H}(\mu)$ which gives a bijective proof of Theorem 1.2. A bijective proof of Theorem 1.1 is given in [10].

We note that Dyck tiles are also used in [1] as a combinatorial tool for Kazhdan-Lusztig polynomials.

\section{Acknowledgement}

I would like to thank Dennis Stanton for drawing my attention to Kenyon and Wilson's conjectures, and for helpful discussion. I also thank Matjaž Konvalinka, David Wilson, and Victor Reiner for helpful comments. 


\section{References}

[1] F. Brenti. Kazhdan-Lusztig and $R$-polynomials, Young's lattice, and Dyck partitions. Pacific $J$. Math., 207(2):257-286, 2002.

[2] J. Cigler and J. Zeng. A curious $q$-analogue of Hermite polynomials. J. Combin. Theory Ser. A, 118(1):9-26, 2011.

[3] P. Flajolet. On congruences and continued fractions for some classical combinatorial quantities. Discrete Math., 41(2):145-153, 1982.

[4] M. E. H. Ismail and D. Stanton. More orthogonal polynomials as moments. In Mathematical essays in honor of Gian-Carlo Rota (Cambridge, MA, 1996), volume 161 of Progr. Math., pages 377-396. Birkhäuser Boston, Boston, MA, 1998.

[5] M. Josuat-Vergès and J. S. Kim. Touchard-Riordan formulas, T-fractions, and Jacobi's triple product identity. http://arxiv.org/abs/1101.5608.

[6] M. Josuat-Vergès and M. Rubey. Crossings, Motzkin paths and moments. Discrete Math., 311:20642078, 2011.

[7] R. W. Kenyon and D. B. Wilson. Boundary partitions in trees and dimers. Trans. Amer. Math. Soc., 363:1325-1364, 2011.

[8] R. W. Kenyon and D. B. Wilson. Double-dimer pairings and skew Young diagrams. Electron. J. Combin., 18:\#P130, 2011.

[9] J. S. Kim. Proofs of two conjectures of Kenyon and Wilson on Dyck tilings. http://arxiv. org/abs/1108.5558

[10] J. S. Kim, K. Mészáros, G. Panova, and D. B. Wilson. Dyck tilings, linear extensions, descents, and inversions. http://arxiv.org/abs/1205.6578

[11] J.-G. Penaud. Une preuve bijective d'une formule de Touchard-Riordan. Discrete Math., 139:347$360,1995$.

[12] H. Prodinger. On Touchard's continued fraction and extensions: combinatorics-free, self-contained proofs. http://arxiv.org/abs/1102.5186.

[13] J. Riordan. The distribution of crossings of chords joining pairs of $2 n$ points on a circle. Math. Comp., 29:215-222, 1975. Collection of articles dedicated to Derrick Henry Lehmer on the occasion of his seventieth birthday.

[14] R. Simion and D. Stanton. Octabasic Laguerre polynomials and permutation statistics. J. Comput. Appl. Math., 68(1-2):297-329, 1996.

[15] J. Touchard. Sur un problème de configurations et sur les fractions continues. Canadian J. Math., 4:2-25, 1952.

[16] G. Viennot. Une théorie combinatoire des polynômes orthogonaux. Lecture Notes, UQAM, 1983. 
[17] G. Viennot. A combinatorial theory for general orthogonal polynomials with extensions and applications. In Orthogonal polynomials and applications (Bar-le-Duc, 1984), volume 1171 of Lecture Notes in Math., pages 139-157. Springer, Berlin, 1985. 